\title{
Reflets
}

Revue ontaroise d'intervention sociale et communautaire

\section{Les mémoires de maîtrise en service social à l'Université d'Ottawa}

\author{
Geneviève Allard, Josée Blanchette, Darline Charles Davilma, Jerryne \\ Mahele-Nyota, Gérard Ntitangirageza et Marie-Andrée Prévost
}

Volume 12, numéro 1, 2006

Spiritualité et intervention sociale

URI : https://id.erudit.org/iderudit/013448ar

DOI : https://doi.org/10.7202/013448ar

Aller au sommaire du numéro

Éditeur(s)

Reflets : Revue ontaroise d'intervention sociale et communautaire

ISSN

1203-4576 (imprimé)

1712-8498 (numérique)

Découvrir la revue

Citer ce document

Allard, G., Blanchette, J., Davilma, D. C., Mahele-Nyota, J., Ntitangirageza, G. \& Prévost, M.-A. (2006). Les mémoires de maîtrise en service social à l'Université d'Ottawa. Reflets, 12(1), 208-215. https://doi.org/10.7202/013448ar

Tous droits réservés (C) Reflets : Revue ontaroise d'intervention sociale et communautaire, 2006
Ce document est protégé par la loi sur le droit d'auteur. L'utilisation des services d'Érudit (y compris la reproduction) est assujettie à sa politique d'utilisation que vous pouvez consulter en ligne.

https://apropos.erudit.org/fr/usagers/politique-dutilisation/ 


\title{
La participation des citoyennes d'Ottawa : comment les femmes d'Ottawa s'organisent-elles afin d'influencer les prises de décisions à un niveau municipal?
}

\author{
Geneviève Allard \\ École de service social, Université d'Ottawa
}

Notre mémoire de maitrise propose une étude portant sur la place des femmes dans le processus décisionnel municipal au sein de la ville d'Ottawa.Vivant dans un contexte international favorable à l'intégration les femmes au niveau municipal, nous avons voulu savoir ce que fait le Canada pour inclure les femmes dans le processus de décisions à ce palier du gouvernement. Quels impacts les femmes ont-elles dans ce processus? Comment les femmes s'y prennent-elles pour influencer les décisions municipales? Ce mémoire présente et fait une analyse du projet Initiative : une ville pour toutes les femmes (IVTF). Il s'agit d'un groupe de femmes d'Ottawa qui s'est rassemblé pour former une "coalition " et ainsi tenter d'influencer les décisions municipales. C'est à travers une approche qualitative, en employant l'étude de cas, que nous avons cherché à connaitre les moyens que les femmes se donnent pour faire entendre leur voix. En travaillant en partenariat avec la ville d'Ottawa, la diversité des femmes de la capitale nationale s'est dotée d'outils et de mécanismes pour s'impliquer et influencer les décisions municipales. Certaines femmes de la ville ont pu profiter d'une formation pilote de lobbying et ensuite la mettre en pratique lors du processus budgétaire de 2005. Elles ont su dire aux conseillers et conseillères l'importance de prendre en considération les voix des femmes dans le budget municipal. Grâce à cet exercice tout au long du processus budgétaire, la diversité des femmes de la ville d'Ottawa a regagné son pouvoir et ainsi influencé les prises de décision au sein de la municipalité. 


\title{
L'insoumission : une alternative
}

\author{
Josée Blanchette \\ École de service social, Université d'Ottawa
}

Bien que le travail social soit caractérisé par une pratique polymorphe et diversifiée, il n'en demeure pas moins qu'il est dominé, en pratique, par une approche gestionnaire du social. L'État demande aux travailleuses sociales de réparer des individus déficients et de les adapter aux normes sociales dominantes. Je dénonce la psychologisation des problèmes sociaux au sein de la profession. Nous revendiquons le champ de l'action sociale mais nous travaillons ce social individuellement. Malgré une analyse structurelle des problèmes sociaux, l'État exige des interventions sectorisées, individualisées, psychologisantes, technicisées, procédurales, etc.

Des effets sur la profession s'en suivent. Les professionnelles se questionnement et se sentent souvent impuissantes. Elles cherchent un sens à leur travail car l'organisation actuelle du travail ne permet pas de s'attaquer à la source des difficultés que nous rencontrons avec les usagers et usagères des services. De plus, l'État n'incite pas au travail collectif et les espaces de travail n'autorisent pas les remises en question du système d'aide et d'assistance. Ce dernier est rigide et de nombreuses résistances proviennent simplement de sa complexité administrative. Dans ce contexte organisationnel, le travail social sert bien souvent à gérer les conséquences d'un système activement responsable des inégalités sociales et de la violence qui s'en suit.

Je propose un travail social engagé et rassembleur, inspiré des pratiques alternatives. Pour dépasser les contradictions dans l'exercice de la profession, les écoles de formation ont un rôle important à jouer. Elles doivent offrir des expériences diversifiées en service social afin que les étudiantes puissent faire un réel choix face aux milieux de pratiques. Actuellement, la formation pratique ne fait que préparer des étudiantes à s'insérer et à 
perpétuer les modes d'intervention dominants. Pour favoriser le renouvellement des pratiques en travail social, une des avenues intéressantes est que l'École se propose d'établir des liens entre les étudiantes et les mouvements sociaux. Offrir les moyens aux étudiantes de s'engager socialement permettrait non seulement de sortir les étudiantes de l'isolement qu'entraîne la recherche mais aussi leur permettrait de participer à un mouvement de transformation sociale.

\title{
La spiritualité et les pratiques religieuses chez les personnes âgées immigrantes haïtiennes : l'implication pour l'intervention sociale - Étude exploratoire
}

\author{
Darline Charles Davilma \\ École de service social, Université d'Ottawa
}

Les études qui présentent la situation des personnes âgées immigrantes au pays d'accueil la décrivent en termes de pauvreté, de perte, d'isolement. En identifiant les problèmes d'intégration des personnes âgées immigrantes, ces études se limitent à l'idée que, outre les activités religieuses, ces dernières n'utilisent pas les services dans la communauté, sans pour autant chercher à connaitre la perception de ces personnes sur leur vie sociale. L'objectif principal de cette recherche consiste à comprendre et à expliquer comment la vie spirituelle des personnes âgées immigrantes haïtiennes et leur participation aux activités religieuses leur permettent de donner sens à leur vie en contexte migratoire. Si l'on veut intervenir adéquatement et de façon appropriée dans un contexte interculturel, on doit, comme le souligne Das (1988: 48), " enquêter à la fois sur les systèmes indigènes d'assistance sociale des diverses communautés, c'est-à-dire, l'aide informelle, le système de soutien naturel et les pratiques formelles d'expertise 
propres à leur culture ", surtout celles qui sont radicalement différentes. En ce sens, une méthode exploratoire de type qualitatif, reposant sur des entrevues semi-dirigées auprès de trois femmes âgées haïtiennes, a été privilégiée. L’analyse des circonstances de la migration, de la vie sociale et économique qui prévalent avant et après l'immigration nous permettent de mieux comprendre le parcours de ces aînés. Arrivées à un âge avancé, ces personnes âgées ont en majorité comme seul point de référence la culture d'origine. Elles restent ancrées dans leur culture et continueront quoi qu'il en soit à interagir à partir d'elle. L'analyse de leur vie spirituelle et de leurs pratiques religieuses permet de dégager les changements qui opèrent à travers l'immigration. La spiritualité se vit au quotidien chez les personnes âgées haïtiennes. La spiritualité et les pratiques religieuses constituent des éléments essentiels dans la vie des personnes âgées haïtiennes en situation de migration. L'appartenance au culte joue un rôle social, de soutien interpersonnel dans la vie et la quotidienneté de ces personnes âgées. Cette appartenance au culte leur permet de garder leur identité comme " haïtienne" par l'usage de leur langue, « le créole » et de leur tradition, en même temps d'assurer une continuité à travers les changements de la vie et l'expérience de la migration. La spiritualité et la participation aux activités religieuses favorisent une meilleure santé subjective, améliorent le fonctionnement physique et permettent aux aînés de trouver un sens à leur vie, à leur souffrance et à la mort. 


\title{
Autre manière de voir, autre manière d'intervenir : enquête exploratoire auprès des organismes offrant des services à des familles immigrantes et réfugiées dont un enfant a un handicap de développement
}

\author{
Jerryne Mahele-Nyota \\ École de service social, Université d'Ottawa
}

Les dernières vingt années ont vu l'arrivée au Canada et à Ottawa de nombreuses familles immigrantes et réfugiées originaires de toutes les régions du monde. Ces familles, dont les croyances et les pratiques dans le domaine de la santé different de celles de la société canadienne, ont à affronter de multiples défis pour s'intégrer à leur société d'accueil. Leur intégration est d'autant plus fragilisée lorsqu'elles ont un enfant vivant avec un handicap de développement. Telle est, du moins, l'hypothèse, que nous avons formulée au départ de cette recherche.

L'objectif de l'étude exploratoire menée pour ce mémoire a été de documenter, d'une part, les difficultés vécues par ces familles immigrantes et réfugiées dont un enfant a un handicap de développement, et d'autre part, ce que font les organismes d'Ottawa qui les aident pour adapter leurs services aux besoins spécifiques de ces familles. Pour cela nous avons mené des entrevues semi-dirigées auprès de huit responsables d'organisme.

L'analyse de ces entrevues nous a permis d'identifier plusieurs types d'obstacles auxquels font face les familles : des obstacles dus au processus d'immigration et d'intégration, des obstacles créés par l'isolement, des difficultés de communication. Cependant, il nous semble que ce sont les différences de perception et de définition du handicap de développement entre parents et intervenants qui entraînent de nombreux malentendus culturels, impliquant la nécessité de repenser l'organisation des services et les modes d'intervention. 
Les intervenantes interrogées ont conscience de ces obstacles et ont proposé des solutions qui ont déjà été évoquées dans de nombreux ouvrages sur la compétence culturelle dans les services sociaux et de santé. Cependant, afin de nous assurer que la défense des droits des familles soit mise de l'avant, nous recommandons en conclusion, la création d'un centre d'information et d'action travaillant avec les parents immigrants et réfugiés qui ont un enfant vivant avec un handicap et évoquons les implications pour l'intervention et les recherches futures.

\section{Jeunes immigrants récemment arrivés au Canada : perceptions qu'ils ont de leur intégration, surtout en milieu scolaire}

Gérard Ntitangirageza

École de service social, Université d'Ottawa

Chaque année, le Canada se donne la mission d'accueillir un nombre important de nouveaux immigrants. Ainsi, la mise sur pied des processus d'intégration efficaces pour ces derniers ne cesse de préoccuper les gouvernements en place et les institutions locales. De leur côté, les nouveaux arrivants doivent développer des stratégies d'intégration pour réaliser leurs projets et pour rencontrer les attentes de la société d'accueil.

Dans la présente étude, nous avons voulu nous intéresser à l'intégration des jeunes immigrants et immigrantes récemment arrivés au Canada, particulièrement en milieu scolaire francophone. Les entrevues réalisées auprès de nos répondants avaient pour but de connaître leurs points de vue quant aux facteurs facilitant ou faisant obstacle à leur intégration.

À la lumière de leurs témoignages, il ressort que le processus d'intégration des nouveaux arrivants dans la société canadienne en général, et en milieu scolaire en particulier, est fortement 
marqué par un effet inégalitaire lié à plusieurs facteurs. Les plus déterminants qui se dégagent de cette recherche sont les chocs de l'arrivée, la qualité de l'accueil tant au niveau du système canadien que du système scolaire, la façon dont les enseignants s'acquittent de leur rôle, le soutien familial, ainsi que les stratégies d'intégration développées par les jeunes eux-mêmes, surtout celles relatives à la maîtrise du français comme langue d'enseignement et de l'anglais qui est la langue essentielle en dehors des salles de classe. Enfin, pour faciliter leur intégration dans la société canadienne et en milieu scolaire en particulier, les participants proposent des changements qui devraient être envisagés.

\title{
L'entraide, l'aide par les pairs et l'aide mutuelle... Regard sur la place de ces types d'aide au sein du système de justice pénale canadien ainsi que dans le processus de réinsertion sociale des ex-détenus
}

\author{
Marie-Andrée Prévost \\ École de service social, Université d'Ottawa
}

Le système de justice pénale au Canada a sous surveillance de nombreux contrevenants et contrevenantes. Beaucoup de ces gens vivent leur quotidien en milieu carcéral ou encore sous surveillance dans la communauté. Pour les gens qui n'ont pas de démêlés avec la justice, le domaine de la criminalité reste un milieu énigmatique : ils en entendent parler, ils en sont des victimes, ou encore, ils la craignent. Dans ce contexte, on se demande s'il pourrait y exister des moyens de rechange qui pourraient arriver à optimiser le bien-être de tous en ce qui a trait à la criminalité et ce qu'en fait le système de justice pénale.

Ce mémoire a donc comme objectif d'offrir des renseignements sur l'historique du crime et de la punition, sur la population carcérale en général, sur les différents services mis sur pied au 
Canada et dans d'autres pays, qui visent à aider les gens ayant un passé carcéral et qui anticipent un retour dans la communauté et finalement, sur les initiatives à promouvoir afin de venir en aide aux gens aux prises avec le système de justice pénal et les moyens à privilégier afin de minimiser certains effets pervers de ce système. À cet égard, nous nous sommes concentrée sur un service, OptionVie qui fut mis sur pied spécifiquement pour les gens condamnés à vie. Ce service est basé principalement sur l'aide par les pairs, mais se réfere également à l'entraide et à l'aide mutuelle, autres notions importantes qui composent le mémoire.

Dans cet ordre d'idée, un regard plus approfondi est porté sur les notions de l'aide par les pairs, de l'entraide et de l'aide mutuelle pour en énoncer les caractéristiques et suggérer les liens possibles à faire avec l'aide professionnelle. De cette façon, nous souhaitons pouvoir établir certains liens en ce qui a trait à ces trois notions et au potentiel qu'elles ont d'influencer de façon bénéfique, à travers différents services, le retour des ex-prisonniers dans la communauté. 